\title{
Probabilistic Design of Optical Transmission Systems
}

\author{
Hou-Man Chin, Douglas Charlton, Andrzej Borowiec, \\ Michael Reimer, Member IEEE, Charles Laperle, Member IEEE, \\ Maurice O'Sullivan and Seb J. Savory, Fellow IEEE, Fellow OSA
}

\begin{abstract}
Traditionally optical fiber nonlinearity is considered a limiting factor for transmission systems. Nevertheless from a system design perspective this nonlinearity can be exploited to minimize the impact of uncertainty on the system performance. A consequence of this is that it becomes beneficial to consider the uncertainty at the design stage, resulting in a probabilistic design, rather than conventional design approaches whereby uncertainty is added by way of system margins to a deterministic design. In this paper we conduct extensive experimental measurements to quantify the impact of uncertainty for a multi-span wavelength division multiplexed (WDM) system transmitting $100 \mathrm{GbE}$ or 200 GbE as dual polarization quadrature phase shift keying (QPSK) or 16 quadrature amplitude modulation (16QAM), respectively. The impact of uncertainty in the power launched into a span is assessed for a $10 \times 80 \mathrm{~km}$ link. For dual polarization (DP)-QPSK the intra-link power deviation with the probabilistic design with $100 \%$ reliability is $\pm 1.3 \mathrm{~dB}$ falling to $99 \%$ reliability with \pm 1.6 dB. In contrast for DP-16QAM maximum deviation for $100 \%$ reliability is $\pm 0.5 \mathrm{~dB}$ falling to $99 \%$ for $\pm 0.6 \mathrm{~dB}$. Following this we consider the interplay between polarization dependent loss (PDL) and fiber nonlinearity over an $8 \times 80 \mathrm{~km}$ system again for both DP-QPSK and DP-16QAM. A system $Q$ variation of less than $0.15 \mathrm{~dB}$ due to the interaction between PDL and fiber nonlinearity is observed for $\mathbf{9 9 . 9 \%}$ of examined PDL values for DP-QPSK and DP-16QAM, thereby allowing the two effects to be considered separately.
\end{abstract}

Index Terms-Optical fiber communication, Systems analysis and design.

\section{INTRODUCTION}

$\mathbf{P}$ ROBABILISTIC design is a methodology based on taking into account the statistical variations of the constituent elements of a system at the design stage, rather than formulating a deterministic design and then considering statistical variations. The probabilistic design framework is particularly beneficial when one is concerned with quantifying the reliability of a design, either to identify the most reliable or more generally, the design that is reliable enough for its purpose. As such, its origins lie in other fields of engineering such as structural and mechanical design where quantifying reliability

Manuscript received July 20, 2016; revised October 02, 2016; accepted December 09, 2016. This research was performed under the auspices of a Ciena university collaborative research grant, with additional support from the RAEng/The Leverhulme Trust and the EU ICONE project grant \#608099.

H-M. Chin is with Orange Polska, Al. Jerozolimskie 160, 02-326 Warsaw, Poland.

D. Charlton, A. Borowiec, M. Reimer, C. Laperle and M. O'Sullivan are with Ciena Corporation, 3500 Carling Avenue, Ottawa ON, K2H 8E9, Canada

S.J. Savory is with the University of Cambridge, Department of Engineering, Electrical Engineering Division, 9 JJ Thomson Avenue, Cambridge, CB3 0FA, U.K. email: sjs1001@cam.ac.uk is extremely important to ensure that random variations do not pose a risk to the design. These variations are modeled as probability distributions whose variances are then summed to generate a joint distribution of overall system performance. Prior to the introduction of probabilistic design, the established means of ensuring that a design would not fail was to apply a 'safety factor'. The safety factor $S$ may be defined as

$$
\mathrm{S}=\frac{\text { Failure Criterion }}{\text { Design Criterion }}
$$

and as such results in a design that is over-engineered for typical operation with typical values of $\mathrm{S}$ ranging from 1.5 (aircraft around the 1940s [1]) to 2 for buildings. While it may seem counter-intuitive that an aircraft had lower safety factor than a building, over-engineering in aircraft results in a heavier design having a greater impact on its engineering and commercial viability than for buildings. In an optical transmission system, the safety factor relates to system margin.

This paper is laid out in the following manner. Firstly the role of systems margin and fiber nonlinearity is examined with respect to fiber-optic transmission systems in light of effects on system performance such as aging. In addition this section briefly introduces two approaches for system design - deterministic and probabilistic design methodologies. These approaches are used to account for various effects that impact system performance over its operational lifetime. Secondly, probabilistic design is further examined. A theoretical fitting model based on the Gaussian Noise (GN) model [2] is introduced to provide ease of examination for an optical transmission system with uncertainty within its optical power profile. This model is verified with extensive measurements using an DWDM experimental setup implementing commercially available coherent transceivers transmitting at 100 and 200 Gbit/s. A heuristic fitting model is introduced to examine the outage probabilities of a transmission link with such intralink power uncertainty in several regimes. The aforementioned theoretical fitting model is then used to examine system behavior for DP-QPSK and DP-16QAM over a wide range of optical power perturbations per fiber span. Lastly the impact of distributed PDL is investigated using a very large number of experimental measurements on a coherent transmission system transmitting DP-QPSK or DP-16QAM. These measurements are then used in conjunction with the prior measurements of a power perturbed optical system to examine the performance of a hypothetical system with both optical power uncertainty in 
addition to distributed PDL allowing for design of the system to a desired outage probability tolerance.

\section{MARGIN AND FIBER NONLINEARITY}

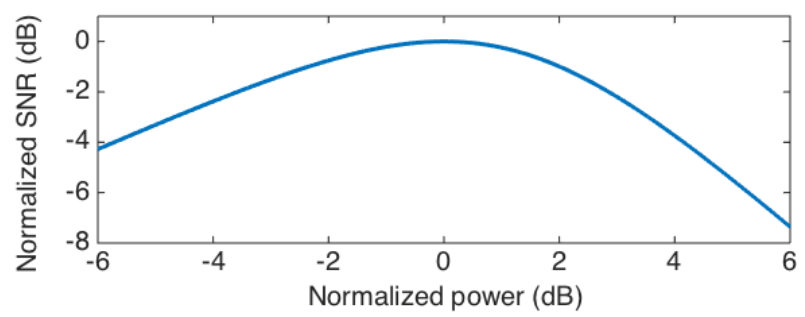

Figure 1. Normalized SNR versus normalized launch power (normalized to the SNR at the optimum launch power and to the optimum launch power respectively)

It is well established that fiber nonlinearities play a significant role in determining the maximum performance of an optical fiber transmission system [3]. The typical effect of optical fiber nonlinearity for a transmission system is illustrated in Fig. 1, where the launch power is normalized to the optimum and the signal-to-noise (SNR) is also normalized to the maximum available (at the optimum launch power). Increasing the transmitted power does not monotonically increase the SNR of the signal. As such the nonlinearity of the fiber presents a considerable detriment to increasing the SNR by increasing the launch power. Nevertheless from the perspective of robust systems, this nonlinearity can be exploited to minimize the impact of variations on the performance such those due to aging which deteriorate the performance of system components. For example if the transmitted power decreases by $3 \mathrm{~dB}$ due to aging over its lifetime, the overall impact on system performance will change greatly depending on the provisioning. As can be seen in table I, due to the fiber nonlinearity there is a significant interaction between the initial launch power $P_{\text {init }}$ and the variation in SNR over life $\Delta S N R=\left|S N R_{\text {init }}-S N R_{\text {final }}\right|$ as the power reduces from $P_{\text {init }}$ to $P_{\text {init }}-3 \mathrm{~dB}$.

\begin{tabular}{|c|c|c|c|}
\hline$P_{\text {init }}(\mathrm{dB})$ & $S N R_{\text {init }}(\mathrm{dB})$ & $S N R_{\text {final }}(\mathrm{dB})$ & $\Delta S N R(\mathrm{~dB})$ \\
\hline-3 & -1.5 & -4.3 & 2.8 \\
\hline 0 & 0 & -1.5 & 1.5 \\
\hline+3 & -2.2 & 0 & 2.2 \\
\hline+1.4 & -0.5 & -0.5 & 0 \\
\hline
\end{tabular}

Table I

IMPACT OF PROVISIONING WITH THE NONLINEAR PERFORMANCE FROM FIG. 1 ON SNR OVER LIFE WITH A 3 dB DEGRADATION IN POWER

As revealed by the last entry in table I, to minimize the transmitted variation with respect to the $3 \mathrm{~dB}$ aging margin, the solution is to deploy at $P_{\text {init }}=1.4 \mathrm{~dB}, S N R_{\text {init }}=$ $0.5 \mathrm{~dB}$, this corresponds to an end of life of $P_{\text {final }}=-1.6$ $\mathrm{dB}, S N R_{\text {final }}=-0.5 \mathrm{~dB}$ with a transmitted variation of 0.5 $\mathrm{dB}$ over its lifetime. Hence by considering the nonlinearity in conjunction with the system margin the transmitted variation is significantly reduced, in this case the $3 \mathrm{~dB}$ uncertainty in power results in a $\pm 0.5 \mathrm{~dB}$ uncertainty in system performance. This example aims to highlight the value of a probabilistic design philosophy whereby uncertainty is considered at the design stage.

The concept of probabilistic versus deterministic systems design is well established, detailed in both standards [4][5] and technical literature [6][7][8]. While probabilistic design has been explored in optical communications, its use has primarily been applied at the components level [9] rather than at a systems level. Nevertheless statistical approaches have been employed for the design of multi-span systems accounting for polarization mode dispersion (PMD) or PDL, with the statistical design resulting in a linear summation of the variances. Of these two, the impact of PDL distributed throughout an optical transmission link is examined in the latter part of this work. The work in this paper builds on our previous work which demonstrated the beneficial effect of fiber optical nonlinearity in managing the impact of uncertainties in an optical system within a probabilistic design framework [10][11].

Current optical fiber communication system design requires that there be sufficient margin to withstand the effects such as aging and system parameter uncertainties. In future networks this margin will also have to absorb the impact of increased network dynamics. This work investigates a probabilistic design approach as an alternative to deterministic design in optical telecommunications systems, accounting for uncertainty at the design stage to minimize its impact on overall system performance.

\section{Probabilistic Design With INTRA-Link POWER DEVIATIONS}

One aspect of an optical link that may prove to be a great influence on its performance are the design tolerances of its constituent elements such as the optical amplifiers. Hence this section examines the effect of changes in the optical power of the signal as it propagates. The system with intra-link optical power uncertainty is implemented as in Fig. 2. The optical power input to the span is perturbed by a fixed positive or negative power deviation, denoted by a 1 and 0 respectively to form a $\mathrm{N}$ bit binary word for a $\mathrm{N}$ span system, for which performance is measured. The power profile of the optical signal with respect to spans is therefore a random walk.

\section{A. Theoretical Fitting Model}

Previously, probabilistic design was demonstrated by the authors in [10][11] in a 10-span fiber transmission system using a single DP-QPSK test channel. Over 87 thousand measurements were utilized. It is clear that investigation of probabilistic design for a realistic number of fiber spans would require an extreme amount of results, either experimental or simulated. It is desirable to have a model with which to examine such optical links for probabilistic design, in particular if the link extends beyond 10 spans and if a more realistic perturbation is required, necessitating a departure from an equally likely fixed perturbation to a different probability distribution e.g. Gaussian or as shown later in this work Maxwellian. There are analytical models for optical fiber 


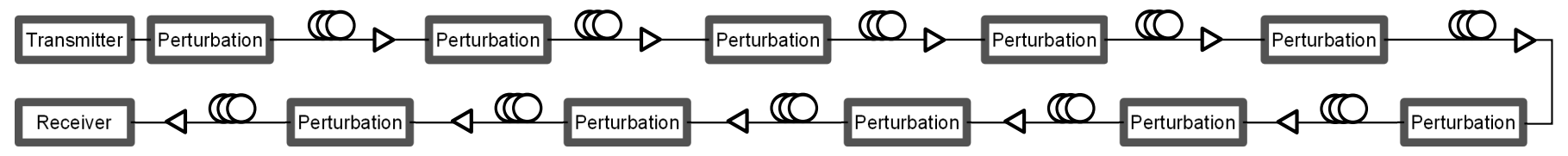

Figure 2. Concept of fiber transmission system with applied optical power perturbation per fiber span

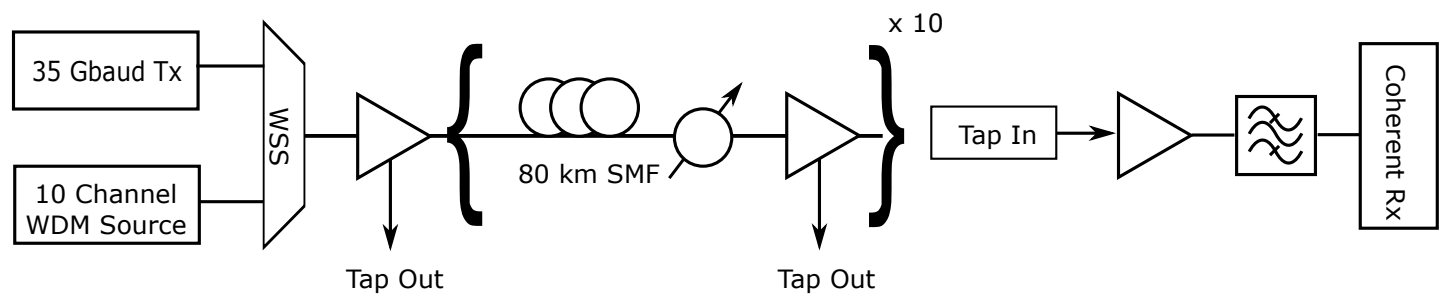

Figure 3. DWDM optical power perturbation experimental setup

propagation, of which the GN model [2] is the most wellknown. These models calculate the power of the nonlinear noise which is then added to amplified spontaneous emission (ASE) noise power to calculate the $S N R$ at the end of the link, from which the BER is calculated.

$$
S N R=\frac{P_{s i g}}{P_{A S E}+P_{N L}}
$$

A three variable model based on the the GN model [2] with which the incoherent accumulation of nonlinear interference per span is assumed. This allows the total link nonlinear noise power to be approximated by summing the nonlinear noise power contribution at each span.

$$
\frac{1}{S N R}=\frac{1}{S N R_{0}}+\sum_{i=1}^{N} \frac{a+b P_{i}^{3}}{P_{i}}
$$

where $S N R_{0}$ is the back to back SNR i.e incorporating power independent effects from the transmitter, receiver and the link itself such as implementation penalty from the digital signal processing (DSP) and additional ASE from transmitter and receiver amplifiers. $N$ is the number of spans, $a$ corresponds to the received amplifier ASE noise, $b$ is the nonlinear interference coefficient such that $P_{N L}=b P_{i}^{3}$ and $P_{i}$ is the optical power into the $i^{t h}$ span. Eqn. 3 was used in [11] to fit the experimental results implying that it is possible to model such a variable system without using computationally expensive split step simulations or experimentally while maintaining a good degree of accuracy. Eqn. 3 is confirmed by further experimental results in a DWDM system below. The values for $a=2.2 \times 10^{-6} \mathrm{~W}$ and $b=6.6 \times 10^{2}$ $\mathrm{W}^{-2}$ obtained experimentally with the unperturbed system are consistent with the GN model for a 10x80 km link with 11x35 GBd channels using the experimental parameters for the fiber $\left(\gamma=1.2 \mathrm{~W}^{-1} \mathrm{~km}^{-1}, D=16.7 \mathrm{ps} \cdot \mathrm{nm}^{-1} \mathrm{~km}^{-1}, \alpha_{d B}=0.19\right.$ $\mathrm{dB} \cdot \mathrm{km}^{-1}$ ) and the amplifiers (gain of $22 \mathrm{~dB}$ with a $5 \mathrm{~dB}$ noise figure). Note that the gain is set higher than the fiber loss per span to compensate for innate loss of in-line optical switches and attenuators as well as providing sufficient optical power for positive power perturbation.

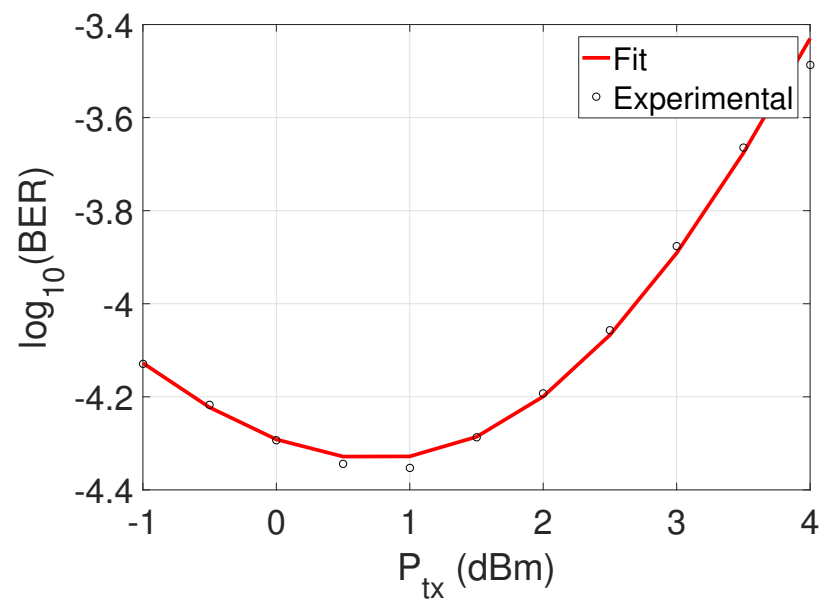

Figure 4. BER performance of DP-QPSK test signal in ideal configuration (markers) with fitted three parameter model (line)

\section{B. Experimental Setup}

The experimental setup was implemented as in Fig. 3. A commercially available 35 Gbaud real-time flexible bit rate transceiver [12] is used to generate a DP-16QAM or DPQPSK test signal at $1547.319 \mathrm{~nm}$. 10 external cavity lasers (ECL) are bulk modulated using a modified version of the test signal transceiver with the same modulation format as the test signal at 35 Gbaud, these channels are then independently optically de-correlated before being recombined using an arrayed waveguide grating (AWG). The de-correlated WDM channels are then combined with the test channel on a $50 \mathrm{GHz}$ frequency grid using a wavelength selective switch (WSS) which also equalizes the channel power. Root raised cosine pulse shaping with $\alpha=0.14$ [12] roll-off is applied to all channels at the transmitters. The transmitter erbium doped fiber amplifier (EDFA) gain is adjusted to provide the desired optical signal power into the first span while the remaining amplifiers are set to constant gain mode with the gain slightly exceeding their respective span losses. The variable optical attenuators (VOA) adjust the loss per span to apply the optical perturbation. The link consists of $10 \times 80 \mathrm{~km}$ spans of standard single mode fiber (SMF). The EDFA after each span has a monitoring 


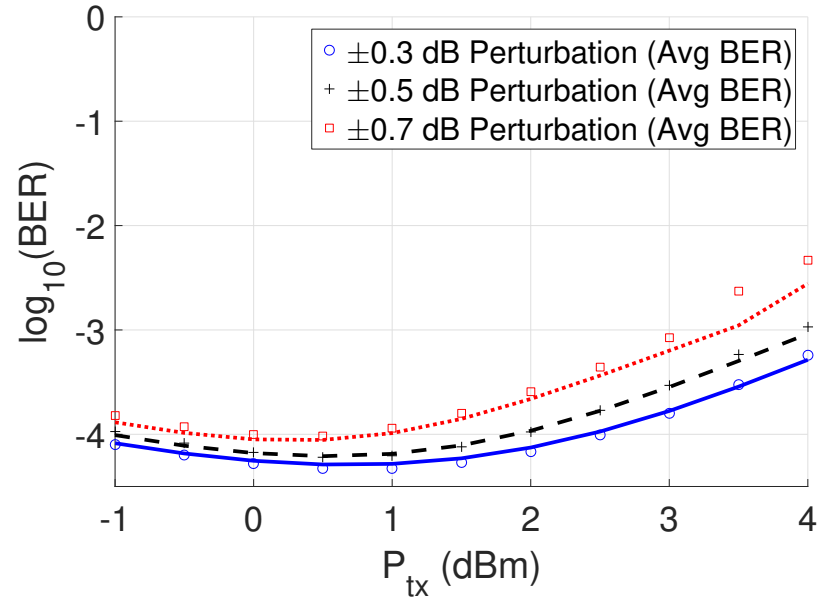

Figure 5. Averaged BER performance for DP-QPSK test channel over 1024 permutations (markers), 3 parameter model (lines)

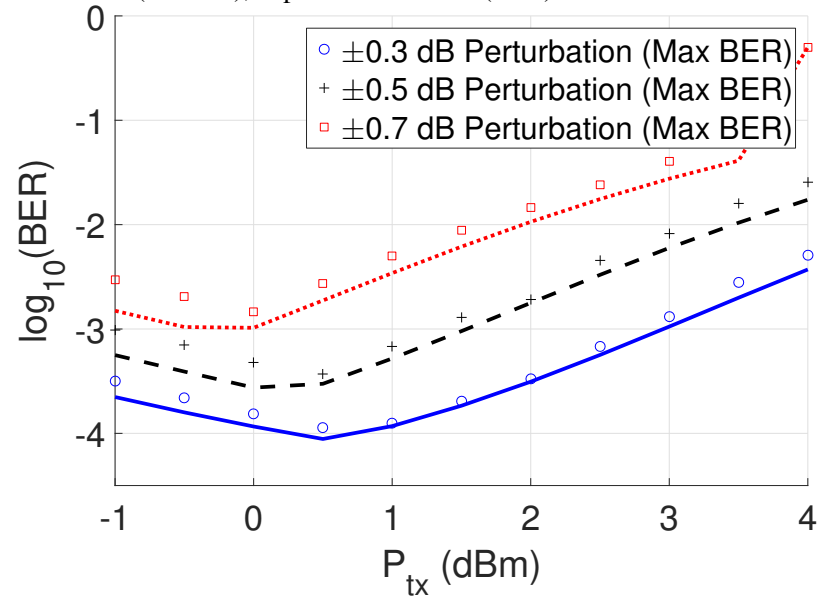

Figure 6. Worst case BER performance for DP-QPSK test channel over 1024 permutations (markers), 3 parameter model (lines)

port which is used to tap out the optical signal which is then band-pass filtered before being received by another real-time coherent modem which processes the received optical signal and measures the bit error rate (BER) before forward error correction (FEC) is applied.

\section{Results}

Fig. 4 shows the performance of the DP-QPSK test signal in the ideal configuration without applied optical perturbations. The three-parameter model is then fitted using a least mean squares method giving parameters $a=2.2 \times 10^{-6} \mathrm{~W}, b=$ $6.6 \times 10^{2} W^{-2}$ and $S N R_{0}=27$ (corresponding to $14.3 \mathrm{~dB}$ ). In the previous single channel experiment, the model was shown to be capable of describing the applied power perturbation. To investigate the predictive capability of the model, a similar set of measurements as before was taken. The perturbation values used are $0.3,0.5$ and $0.7 \mathrm{~dB}$, the average and worst case BER performances are shown in Fig. 5 and Fig. 6 respectively and with good agreement found between that predicted by the model and the average BER performance albeit the model becomes less accurate for the worst case BER performance. A decrease in the optimum launch power is observed with

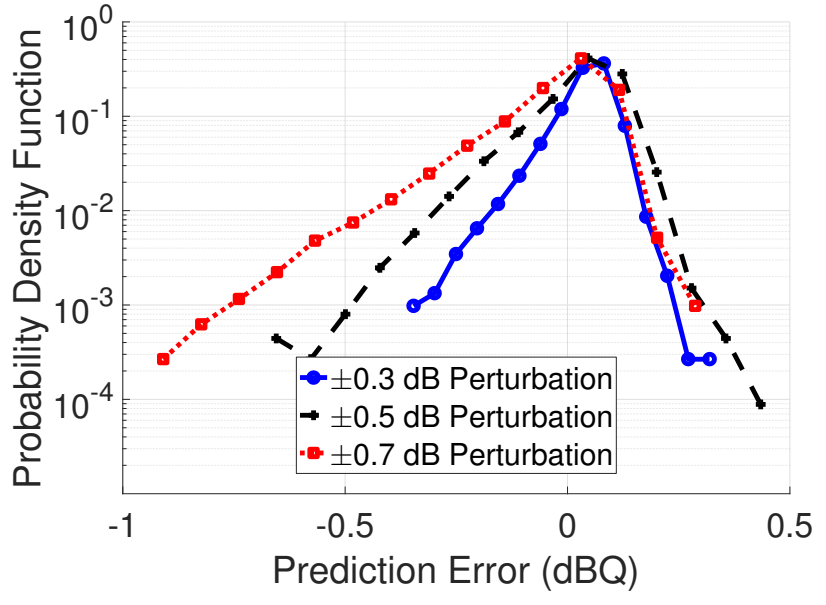

Figure 7. Probability distribution of the error in $\mathrm{dB}(\mathrm{Q})$ for the model prediction with respect to experimental measurement for the DP-QPSK test signal

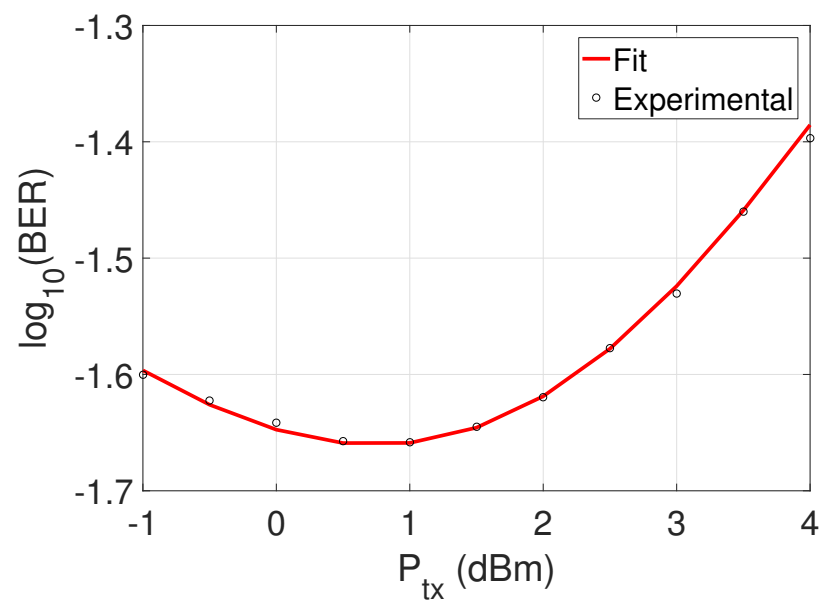

Figure 8. BER performance of DP-16QAM test signal in ideal configuration (markers) with fitted three parameter model (line)

respect to increasing perturbation magnitude. The distribution of the errors is plotted in Fig. 7, with the average absolute error being $0.09 \mathrm{~dB}$ and the maximum observed error being a $0.9 \mathrm{~dB}$ (with $\pm 0.7 \mathrm{~dB}$ perturbations).

The transceivers are switched to modulating DP-16QAM at the same baud and with the same pulse shaping applied. Fig. 8 shows the test channel pre-FEC BER performance in the absence of optical power perturbations and with fitted model. The model parameters are $a=2.2 \times 10^{-6} \mathrm{~W}, b=6.6 \times$ $10^{2} W^{-2}$ and $S N R_{0}=36$ (corresponding to $15.5 \mathrm{~dB}$ ). As with the previous DP-QPSK experiment, the model fits very well to the ideal case. The averaged BER performance shown in Fig. 9 shows good agreement between the model predictions and experimental results, up to the pre-FEC BER threshold of $3.4 \times 10^{-2}$. The change in optimum launch power is again present for DP-16QAM and is predicted by the model. The worst case performance is illustrated in Fig. 10 with again, good agreement between model and experimental results.

The difference between the model DP-16QAM performance prediction and experimental results is shown in Fig. 11 for each perturbation. The error of the prediction is less than in 


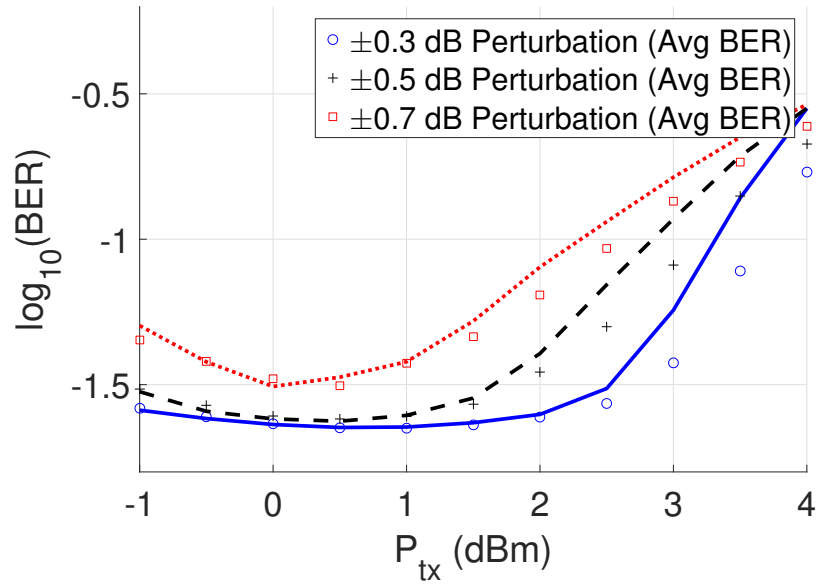

Figure 9. Averaged BER performance for DP-16QAM test channel over 1024 permutations (markers), 3 parameter model (lines)

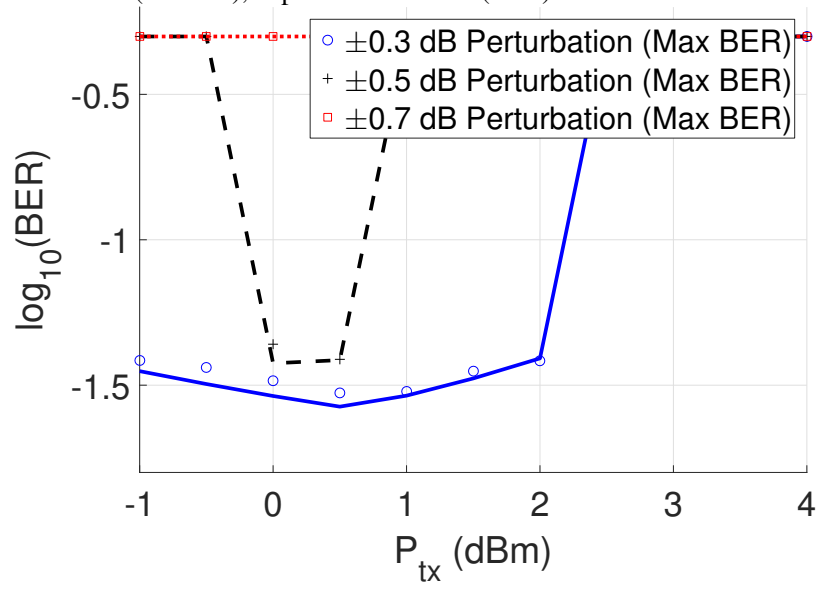

Figure 10. Worst case BER performance for DP-16QAM test channel over 1024 permutations (markers), 3 parameter model (lines)

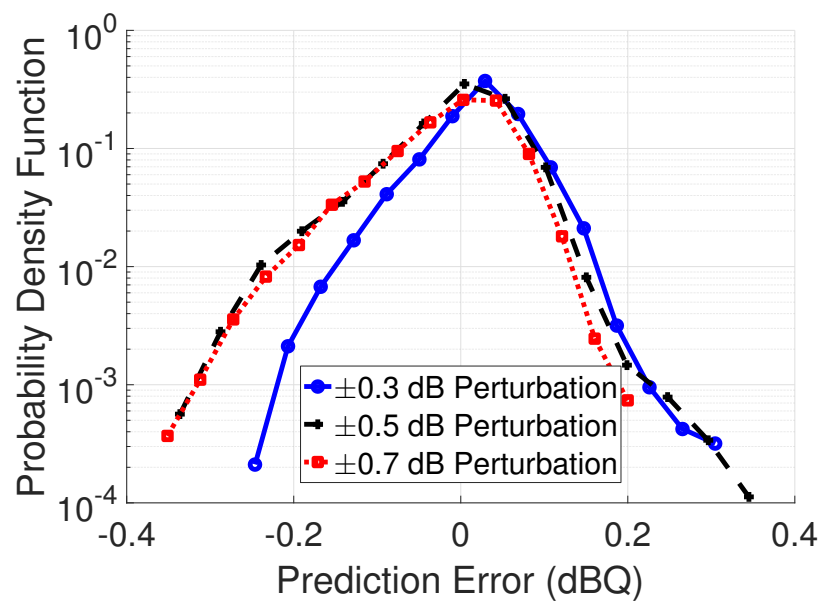

Figure 11. Probability distribution of the error in $\mathrm{dB}(\mathrm{Q})$ for the model prediction with respect to experimental measurement for the DP-16QAM test signal the DP-QPSK comparison since the impact of the nonlinear distortion is modulation format dependent [13] which is unaccounted for in this model. DP-16QAM has been shown to be more accurate with the standard GN model. [2] The largest error is $\approx 0.4 \mathrm{~dB}(\mathrm{Q})$ overestimation of channel performance with a mean absolute error of less than $0.1 \mathrm{~dB}(\mathrm{Q})$.

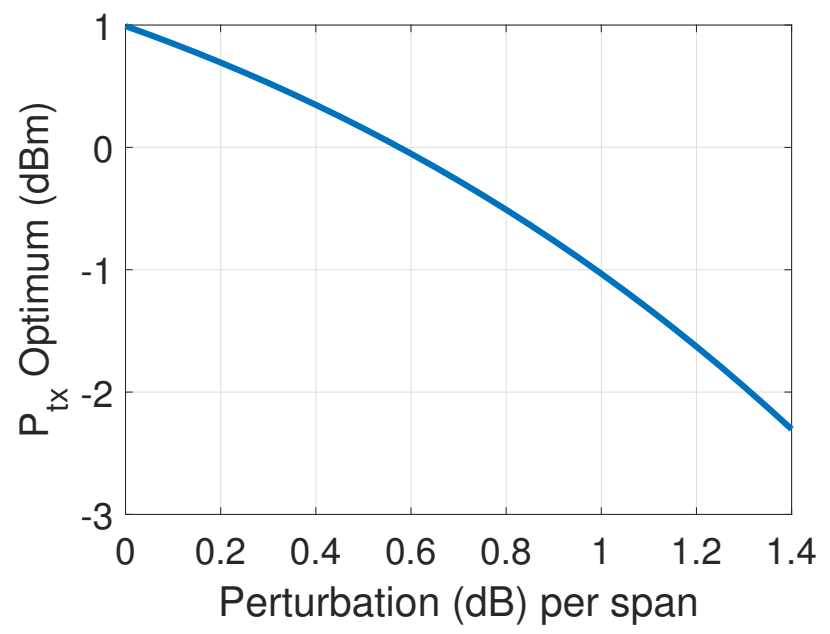

Figure 12. Modeled optimum launch power for DP-QPSK configuration

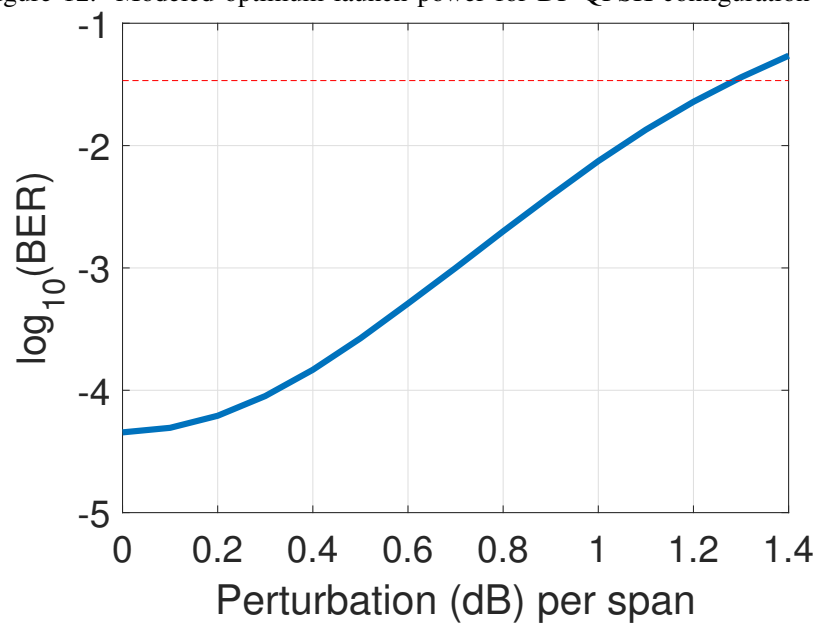

Figure 13. Modeled DP-QPSK worst case BER with respect to increase in perturbation magnitude,SD-FEC threshold shown as dashed line

\section{Application of Results to Probabilistic Design}

Previously [11], the theoretical model was verified with a large number of experimental measurements generated by perturbing an optical transmission system with a limited number of perturbations. Here, this work goes into greater depth on the impact of such intra-link power variations. From a systems design perspective, the provisioning of the optical launch power is extremely important. Using the model, the optimum launch power is calculated for the DWDM transmission system described by Fig. 3 first for DP-QPSK and then for DP16QAM. Fig. 12 shows the evolution of the optimum launch power with the increase in the magnitude of the applied perturbation. Paired with this, is the change in the worst case BER shown in Fig. 13. When the perturbation increases past $1.3 \mathrm{~dB}$, 


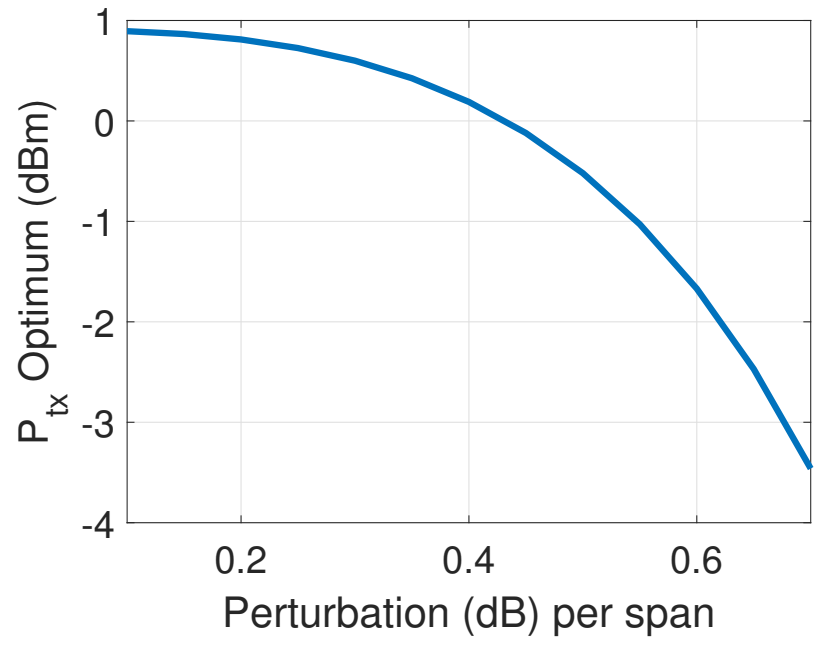

Figure 14. Modeled optimum launch power for DP-16QAM configuration

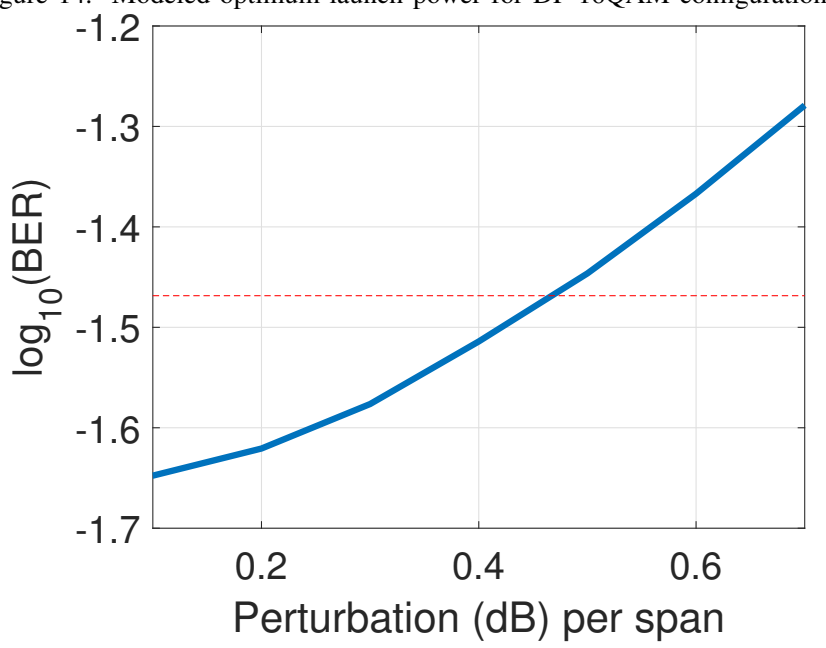

Figure 15. Modeled DP-16QAM worst case BER with respect to increase in perturbation magnitude, SD-FEC threshold shown as dashed line

the worst case performance increases beyond the capability of the SD-FEC to compensate for $\left(\mathrm{BER} \leq 3.4 \times 10^{-2}\right)$, therefore leading to system outage. For a perturbation of $1.4 \mathrm{~dB}, 4$ cases out of 1024 were shown to be outages, giving a reliability of approximately $99.6 \%$. An increase to a $1.6 \mathrm{~dB}$ perturbation reduces link reliability to $99 \%$. In [11], an illustrative example of different provisioning regimes for the launch power was described. There are some interesting design decisions that can be made as a result of this information. If it is decided to maximize system reliability at time of deployment, then obviously provisioning at the optimum launch power is the best deployment, Fig. 12 combined with a cumulative distribution of systems performance for the designed systems tolerance enables this decision. An alternative design decision would be to minimize the variation in systems performance due to time dependent effects (mainly aging) which would decrease the optical power in the link. Launching at a higher power than the optimum would ameliorate this effect at the cost of initial systems performance and an increase in systems outage probability. The third would be a scenario where the designer is forced to accept a system that cannot give each channel sufficient power to achieve the optimum, perhaps due to upgrading a legacy system to a smaller frequency grid and therefore we must examine the system to determine if it will perform with acceptable outage probability.

Fig. 14 shows the modeled optimum launch power and Fig. 15 shows the worst case BER using the parameters extracted from the DP-16QAM experimental measurements with respect to applied perturbation. When the perturbation exceeds $0.5 \mathrm{~dB}$, the worst case BER exceeds the ability of the transceiver to correct. If the reliability requirement can be relaxed for example to $99 \%$ the maximum perturbation can be increased to $0.6 \mathrm{~dB}$.

\section{Probabilistic Design in the Presence of DISTRIBUTED PDL}

\section{A. Background}

Another area that has undergone significant examination is that of the effect of PDL. In [14], PDL was shown to have a probability distribution that is Maxwellian. It has been well established that the bounds of the impact of PDL on coherent transmission systems is delineated by the incident angle of the optical signal to the polarization loss axis of the optical component causing the PDL [15][16][17]. An initial experimental investigation of the effect of distributed PDL on dispersion uncompensated coherent optical transmission systems was shown by us in [18]. The PDL can be described as the following Jones matrix

$$
\mathrm{T}_{\mathrm{PDL}}=\left[\begin{array}{cc}
1 & 0 \\
0 & e^{-\alpha}
\end{array}\right]
$$

where $\mathrm{PDL}_{\mathrm{dB}}=10 \log _{10} \mathrm{e}^{2 \alpha}=20 \alpha \log _{10}(\mathrm{e})$, the incident angle of the optical signal can be described by a rotation matrix $R$

$$
R=\left[\begin{array}{cc}
\cos \theta & \sin \theta \\
-\sin \theta & \cos \theta
\end{array}\right]
$$

where $\theta$ is the angle between one polarization of the optical signal and the polarization loss axis. The local PDL of the element is therefore

$$
\begin{gathered}
\mathrm{H}=\mathrm{T}_{\mathrm{PDL}} \mathrm{R} \\
\mathrm{H}_{\text {worst }}=\mathrm{T}_{\mathrm{PDL}} \mathrm{R}\left(\theta=0^{\circ}\right) \\
\mathrm{H}_{\text {best }}=\mathrm{T}_{\mathrm{PDL}} \mathrm{R}\left(\theta=45^{\circ}\right)
\end{gathered}
$$

The worst and best bounds of PDL are at $\theta=0^{\circ}$ and $\theta=45^{\circ}$ and their effect on the optical signal. At $\theta=0^{\circ}$, there is simply a degradation of SNR in one axis of polarization and the signal's orthogonality is unchanged. When $\theta=45^{\circ}$, both polarizations suffer the same loss in SNR and also a loss in orthogonality between the two polarizations. This is interesting with respect to the above investigations when there is a deviation in power during link propagation since, the optical signal is amplified after propagation through a fiber span according to the average optical power. This means that the power differential between polarizations when $\theta \neq 45^{\circ}$ is preserved. Potentially this can increase the nonlinearity experienced by the system due to the increased optical power in one polarization. If the interaction between PDL and nonlinearity 
is significant, this also increases the difficulty of designing a system since the two input parameters are dependent. Existing work has shown that this may be a challenge [15]. This is exacerbated by the requirement for increased network functionality in next generation networks [19], which necessitate the deployment of optical components such as reconfigurable add drop optical multiplexers (ROADM).

\section{B. Experimental Setup}

This section investigates the interaction of link distributed PDL with fiber nonlinearity in a 63 channel DWDM system using commercial transceivers over an dispersion uncompensated system transmitting over $640 \mathrm{~km}$ of SMF. The experimental setup used for the PDL investigations is as shown in Fig. 16. A commercially available real time transceiver transmitting at 35 Gbaud is used to modulate DP-QPSK or DP16QAM at $1547.319 \mathrm{~nm}$. 62 de-correlated 35 Gbaud signals modulated with the same format as the test signal are copropagated on the ITU $50 \mathrm{GHz}$ grid. The transmitter lasers are ECLs with $20 \mathrm{kHz}$ linewidth. Root raised cosine pulse shaping with a roll off $(\alpha=0.14)$ is applied to all channels. A CW laser with $10 \mathrm{~dB}$ more power is coupled with the test signal and then input to a polarization synthesizer which used the single polarization $\mathrm{CW}$ source as a reference to jointly stabilize both the test and $\mathrm{CW}$ signals. The $\mathrm{CW}$ laser is de-multiplexed by the wavelength selective switch (WSS) which also equalizes the channel transmitted power for maximum flatness at the input to span 5. The fiber link is composed of 8 spans of $80 \mathrm{~km}$ SMF. Each SMF span is followed by a polarization controller and then a $1 \mathrm{~dB}$ PDL element. The optical signal is received by another real time transceiver and the signal is then processed using the on-board DSP. The launch power is incremented from -2 to $3 \mathrm{dBm}$ in $1 \mathrm{~dB}$ steps.

\section{Results}

The input distribution of link PDL instances is generated by randomly setting the polarization controllers to generate 10,000 discrete PDL instances per launch power. The link PDL is extracted from the degree of polarization (DOP) using the relationship derived in the Appendix, namely

$$
\mathrm{PDL}_{\mathrm{dB}}=20 \log _{10}(\mathrm{e}) \times \tanh ^{-1}(\mathrm{DOP})
$$

The link PDL distribution measured using Eqn. 9 is shown in Fig. 17 to be Maxwellian as expected. For each instance of link PDL, the pre-FEC BER is measured and then mapped to $\mathrm{Q}$ using $Q=20 \times \log _{10}\left(\sqrt{2} \times \operatorname{erfc}^{-1}(2 \times B E R)\right)$. Fig. 18 shows the cumulative probability of the impact of link PDL on coherent systems performance. The key measurement of note is that the -2 and $3 \mathrm{dBm}$ distributions are very similar. Across the $5 \mathrm{~dB}$ range of launch powers there is less than $0.14 \mathrm{~dB}$ difference at $10^{-3}$ probability which increases with decrease in probability. Each set of 10,000 link PDL instances were independently generated and is a possible explanation as the the variation between the distributions.

To further emphasize this, Fig. 19 shows the superimposed probability distributions normalized to the mean Q per optical launch power. It can be seen that there is a small amount of interplay between PDL and fiber nonlinearity given the disparity between the $-2 \mathrm{dBm}$ and $3 \mathrm{dBm}$ distributions from the decrease in probability around the peaks however there is very little change in the overall shape of the distributions. To gain a sense of the impact of this on the provisioning of the transmission system, the values of link PDL are binned with $0.5 \mathrm{~dB}$ granularity. The BER values associated with these bins are averaged and their curves fitted and plotted in Fig. 20. Note that the higher PDL bins are not are not fitted due to poor fits as a result of low bin population. A similar conclusion is reached in that the optimum system launch power is essentially unchanged as the link PDL increases. Hence the link PDL and fiber nonlinearity may be treated as independent variables when considering implementation of probabilistic design. The previous experimental measurements are retaken with all DWDM channels set to modulate DP16QAM.

Fig. 21 shows the link PDL distribution over the 60,000 instances generated for the DP-16QAM measurements, similar to the previous 60,000 instances generated for the DP-QPSK measurements it is Maxwellian in shape. Fig. 22 shows the cumulative distributions for each optical launch power. In contrast to the previous DP-QPSK results, there is a slight trend in the change in $\mathrm{Q}$ as the optical launch power is incremented, however across the $5 \mathrm{~dB}$ range of launch powers there is only a spread of $0.15 \mathrm{~dB}$ at $10^{-3}$ probability. Accordingly, it can be seen from Fig. 23 that there is a widening of the probability distributions shown with respect to launch power indicating that for higher values of system PDL (occurring at lower probabilities) there is some interaction with fiber nonlinearity when transmitting DP-16QAM. In absolute terms it is correspondingly small though we note that for the equivalent change in $\mathrm{Q}$ in Fig. 23 at $10^{-3}$ probability with $\mathrm{P}_{\mathrm{tx}}=-2 \mathrm{dBm}$, the probability rises to $4.3 \times 10^{-3}$ for $\mathrm{P}_{\mathrm{tx}}=3$ $\mathrm{dBm}$. Again there is essentially negligible difference in the optimum provisioning shown by the averaged BER values in Fig. 24. Therefore for a dispersion unmanaged DP-16QAM transmission system, the impact of PDL and fiber nonlinearity can also be considered separately. However the fact that there appears to be some dependence of this on the cardinality of the modulation format necessitates investigation of higher order modulation formats before assuming that this similarly holds for them.

To further highlight the system benefit of probabilistic design we consider an illustrative example based on DPQPSK with $\pm 0.7 \mathrm{~dB}$ perturbations per span with distributed link PDL. As can be seen in Fig. 25 which shows the cumulative distribution for a range of optical launch powers the probabilistic design has up to a $1.4 \mathrm{~dB}(\mathrm{Q})$ advantage in performance at less than $10^{-3}$ probability compared to a deterministically designed system operating at the optimum of $+1 \mathrm{dBm}$. While margin could be applied to account for the uncertainty the probabilistic design framework reveals the correct margin to be applied, namely $1 \mathrm{~dB}$ for a received power of $\pm 7 \mathrm{~dB}$ (caused by 10 spans each with $\pm 0.7 \mathrm{~dB}$ ). 


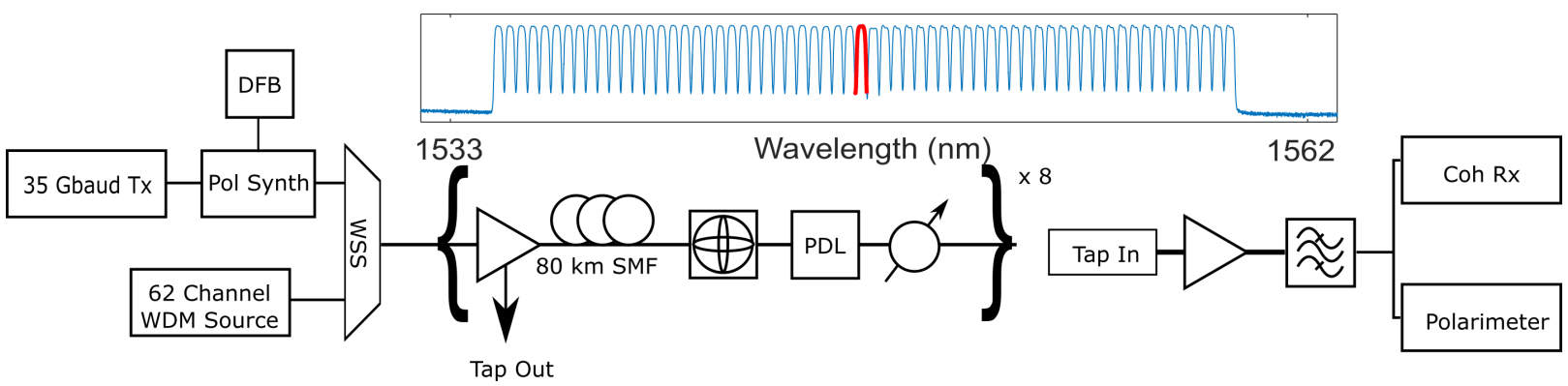

Figure 16. Experimental setup for investigation of distributed PDL

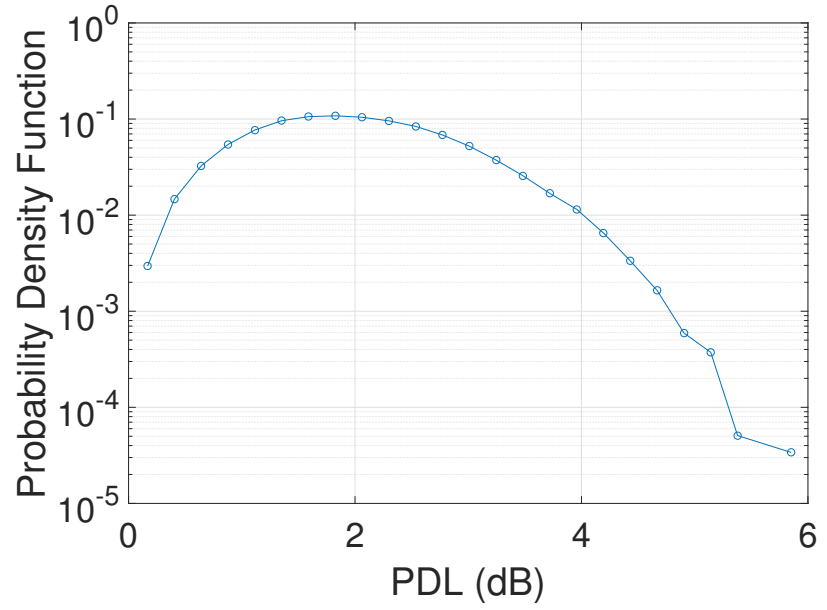

Figure 17. Distribution of 60,000 link PDL instances for DP-QPSK results $(10,000$ for each of the 6 optical launch powers $)$

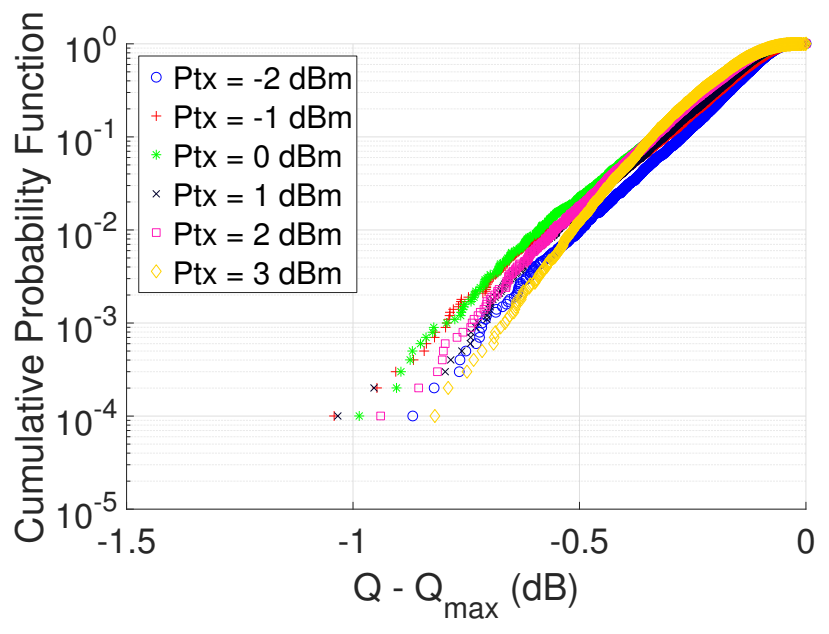

Figure 18. Impact of distributed PDL on DP-QPSK system

\section{CONClusion}

In this work, an alternative to the typical deterministic design approach currently in use for optical transmission systems is explored. The probabilistic design approach has been widely used in other fields of engineering but not applied to designing optical communications links. Inherent to every engineering design is the inability to have absolute knowledge of all design parameters at every point in the design's lifetime. Nor is it

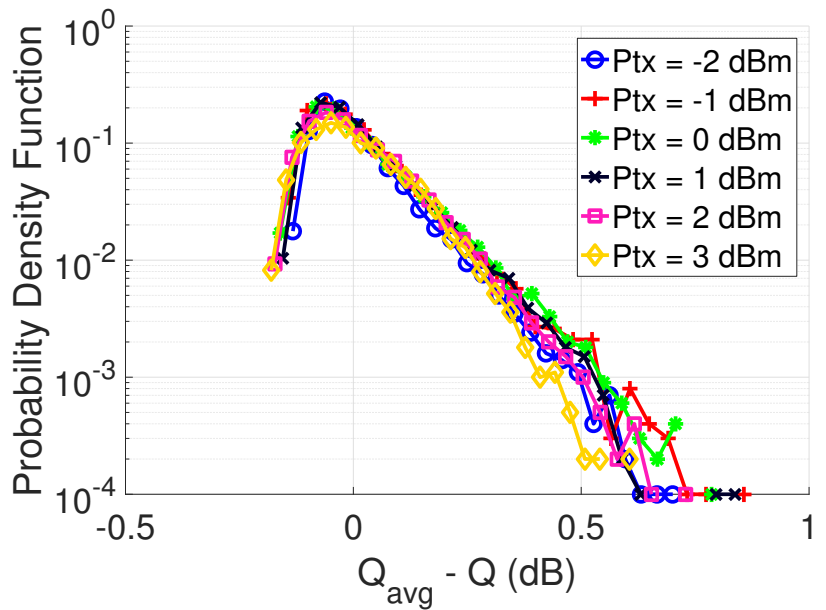

Figure 19. Impact of distributed PDL on DP-QPSK system

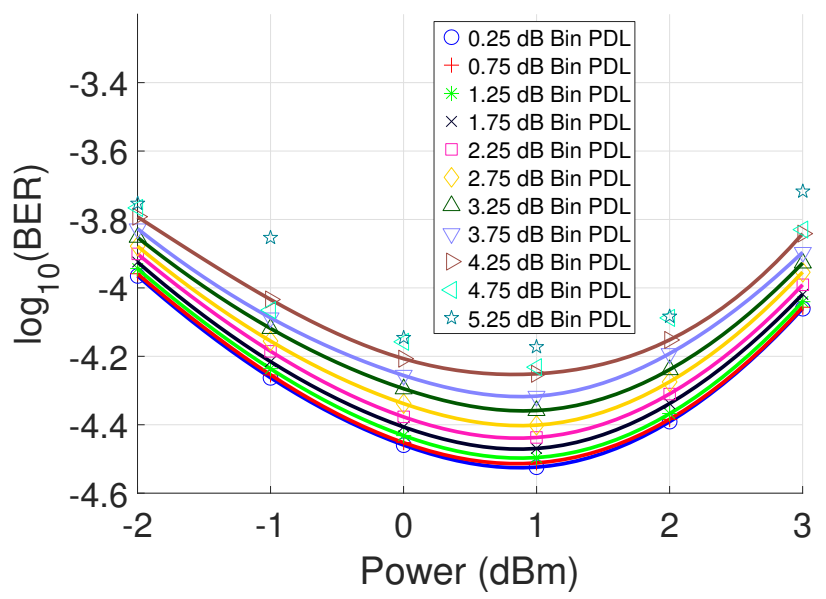

Figure 20. Impact of distributed PDL on DP-QPSK system

possible that every systems' component is made exactly to design specifications. The probabilistic design approach seeks to account for this uncertainty in the design variables to ensure an acceptable degree of performance over the operational life cycle.

The optical fiber transmission medium provides an interesting opportunity due to the nonlinear nature of its silica core. In other systems using a linear transmission medium, such as wireless transmission systems, a probabilistic design approach would simply be to sum the variances of the probability 


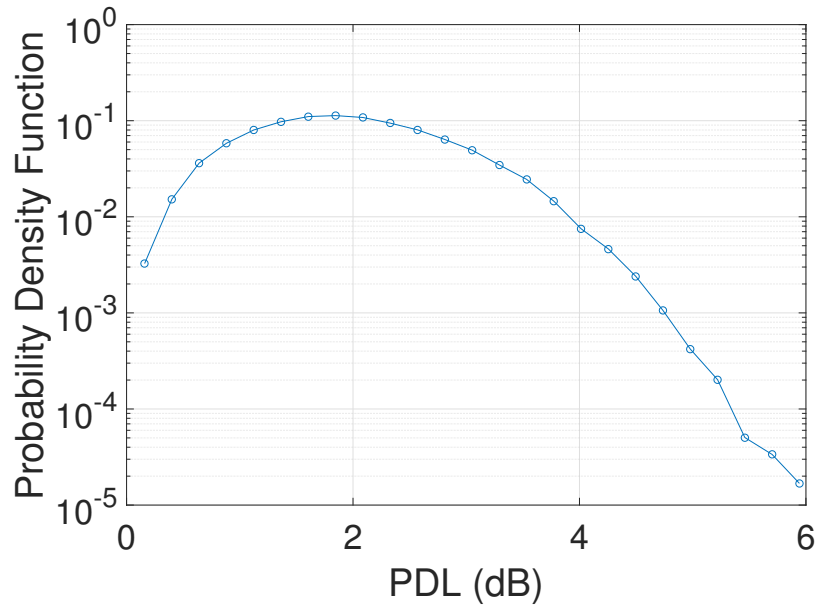

Figure 21. Distribution of 60,000 link PDL instances for DP-16QAM results

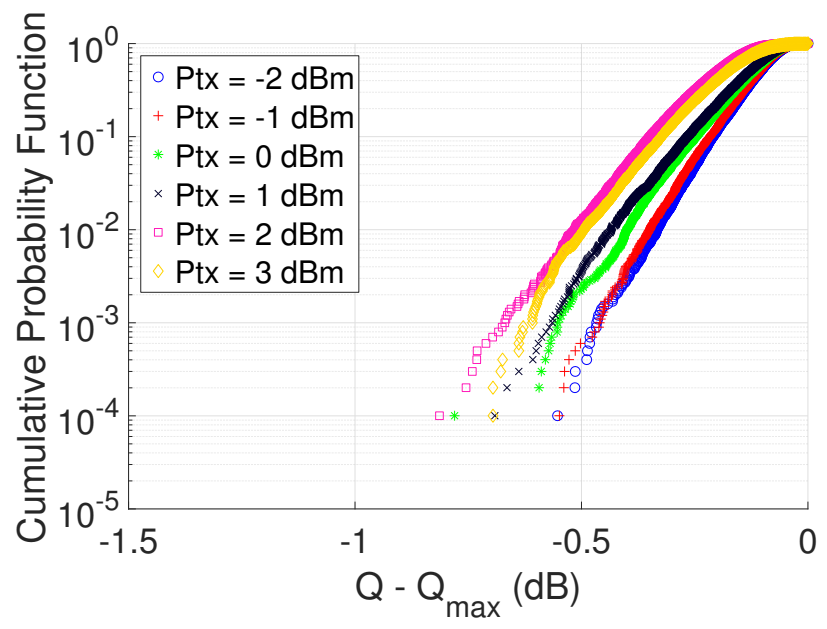

Figure 22. Impact of distributed PDL on DP-16QAM system

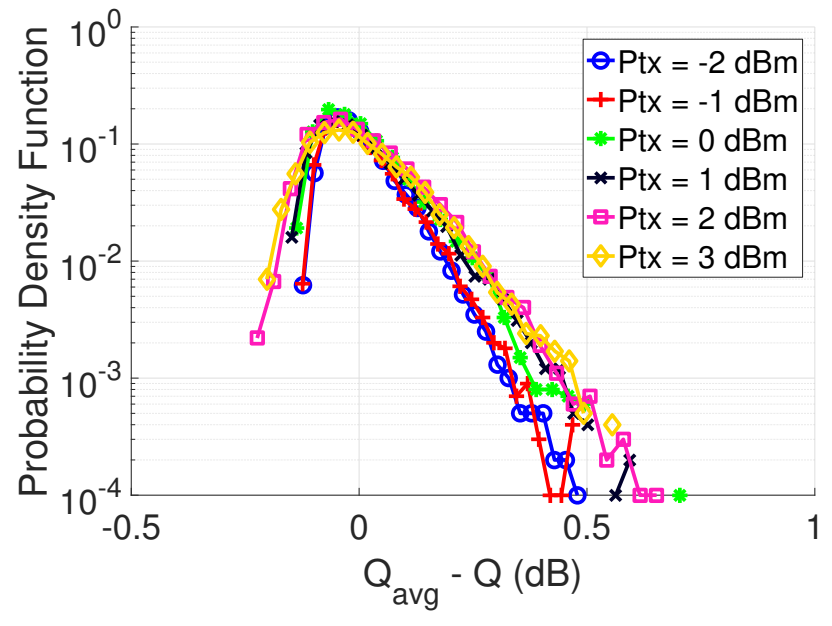

Figure 23. Impact of distributed PDL on DP-16QAM system

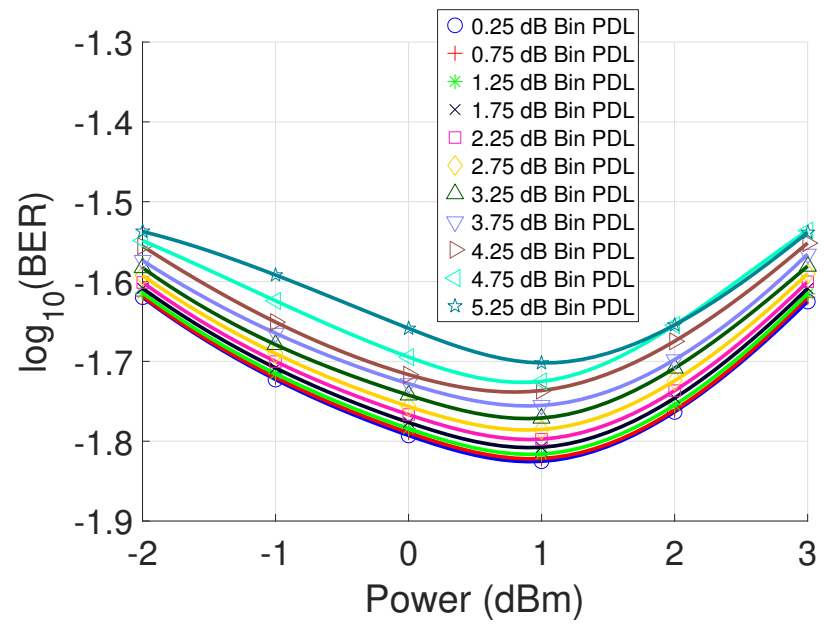

Figure 24. Impact of distributed PDL on DP-16QAM system

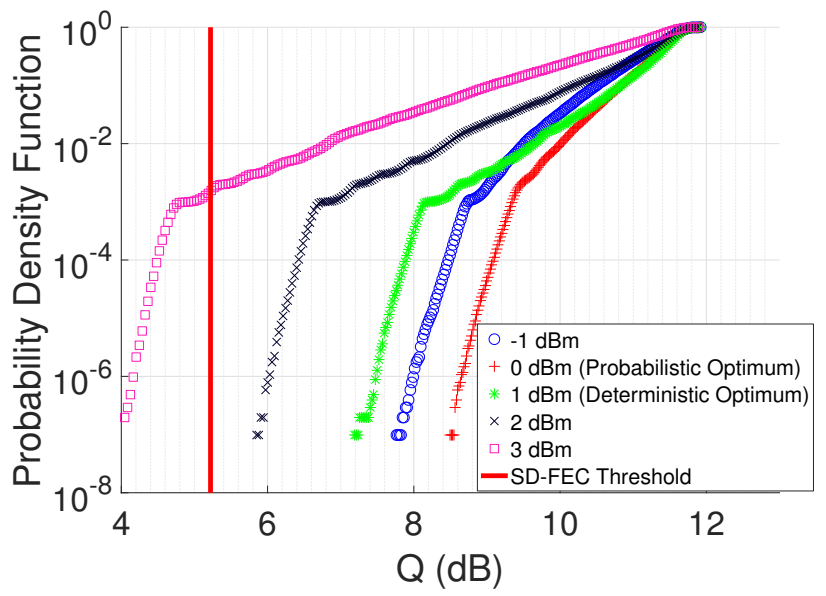

Figure 25. Cumulative distribution for a 10 span $800 \mathrm{~km}$ DP-QPSK system over a range of optical launch powers with $0.7 \mathrm{~dB}$ applied optical power perturbation per span and distributed link PDL. Deterministic and probabilistic design optima are at $P_{t x}=1$ and $0 \mathrm{dBm}$ respectively

distributions of the input design parameters. However, due to fiber nonlinearity, if as in this work there is a uncertainty in the channel optical power during propagation along the fiber, the output probability distribution may not be shaped the same as the input distribution. Therefore by considering this in the design process, it is possible to design a more robust system.

Previous research has shown that implementing probabilistic design to account for systems uncertainty at the design process can be advantageous, both from a performance perspective and also to know the expected reliability of the system. However, even implementing the Bernoulli choice used in these experiments with a $2^{N}$ cases, where $N$ is the number of spans in the fiber link, the number of cases to be investigated increases rapidly. A numerical simulation method such as the split step Fourier method may be possible but would be computationally expensive, hence why this work uses a real-time experimental setup. The number of measurements is limited by the equipment reconfiguration time which is on the order of seconds, this work achieved a measurement every 8 seconds. If a full investigation with Gaussian distributed 
optical power input to each span is required, achieving a 'worst case' or close to it needs a very large number of measurements which would be rather impractical. Therefore, it is desirable to have a fast method investigate such a scenario. This work proposed a three-parameter model based on the GN-model of fiber propagation to address this. The model previously [10] successfully parameterized the performance of the perturbed test channel over the 5 different perturbations used for a single channel 10 span system with the same values.

In this work the probabilistic design was investigated for an 11 channel system based on the ITU $50 \mathrm{GHz}$ frequency grid for DP-QPSK (100 Gbit/s) and DP-16QAM (200 Gbit/s). In a differentiation from the previous work [10], the unperturbed system is used as a reference to extract the parameters for the model. These parameters are then used to estimate the system performance when perturbation is applied. It was found that the model's predictive ability was in generally good agreement with the experimental measurements with an average error of less than $0.1 \mathrm{~dB}$ for both DP-QPSK and DP-16QAM. The optimum transmission regime under perturbative scenarios was also successfully predicted over a $5 \mathrm{~dB}$ power range.

To further extend probabilistic design, PDL elements were introduced throughout the experimental setup to approximate the presence of distributed PDL. 120,000 instances of link PDL were generated in total for DP-QPSK and DP-16QAM measurements $(60,000$ each). It was found that there was almost non-existent interplay between distributed PDL and fiber nonlinearity at high transmission powers. This allowed the impact of optical power perturbations and distributed PDL to be treated as independent. From this a cumulative distribution was generated for a range of launch powers allowing for systems design in the presence of intra-link optical power uncertainty and distributed PDL.

\section{APPENDIX}

In this appendix we derive a relationship between the polarization dependent loss and degree of polarization for a polarization multiplexed signal. We begin by noting that PDL can be represented as

$$
\begin{aligned}
\mathrm{T}_{\mathrm{PDL}} & =e^{-\alpha / 2} e^{(\hat{\alpha} \cdot \vec{\sigma}) \alpha / 2} \\
& =e^{-\alpha / 2}\left[I \cosh \frac{\alpha}{2}+(\hat{\alpha} \cdot \vec{\sigma}) \sinh \frac{\alpha}{2}\right]
\end{aligned}
$$

where $\alpha$ is the PDL coefficient such that $\mathrm{PDL}_{\mathrm{dB}}=$ $10 \log _{10} \mathrm{e}^{2 \alpha}=20 \alpha \log _{10}(\mathrm{e}), \hat{\alpha}$ is the minimum loss axis on the Poincaré sphere, $\mathrm{I}$ is the identity matrix and $\vec{\sigma}$ is the spin vector whose elements are the Pauli matrices.

If we consider a normalized input $|\hat{s}\rangle$ such that $\langle\hat{s} \mid \hat{s}\rangle=$ 1 then the received signal is given by $\mathrm{T}_{\mathrm{PDL}}|\hat{\mathrm{s}}\rangle$. In order to calculate the degree of polarization we calculate the received Stokes vector given by

$$
\mathrm{S}_{\mathrm{rx}}=\left\langle\mathrm{T}_{\mathrm{PDL}} \hat{\mathrm{s}}|\vec{\sigma}| \mathrm{T}_{\mathrm{PDL}} \hat{\mathrm{s}}\right\rangle=\left\langle\hat{\mathrm{s}}\left|\mathrm{T}_{\mathrm{PDL}} \vec{\sigma} \mathrm{T}_{\mathrm{PDL}}\right| \hat{\mathrm{s}}\right\rangle
$$

noting that the following identities apply

$$
\begin{array}{r}
\vec{\sigma}(\hat{\alpha} \cdot \vec{\sigma})+(\hat{\alpha} \cdot \vec{\sigma}) \vec{\sigma}=2 \hat{\alpha} \\
(\hat{\alpha} \cdot \vec{\sigma}) \vec{\sigma}(\hat{\alpha} \cdot \vec{\sigma})=\hat{\alpha} \cdot(\hat{\alpha} \cdot \vec{\sigma})-\vec{\sigma}
\end{array}
$$

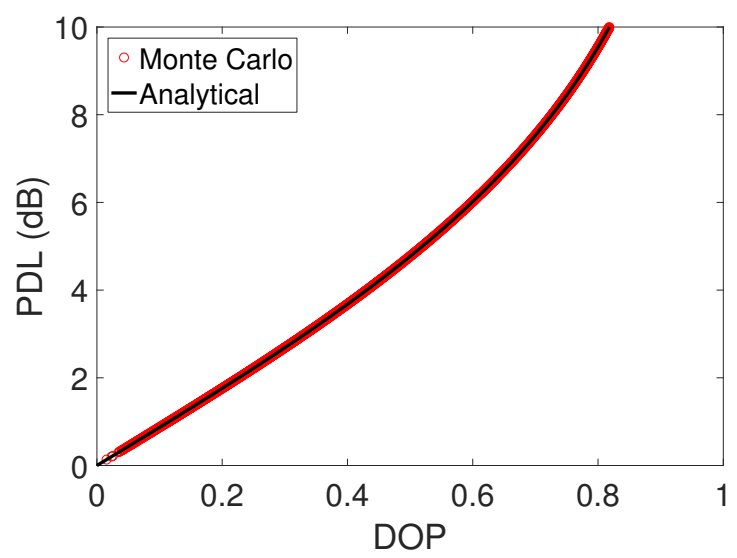

Figure 26. Comparison of analytical expression of DOP vs PDL and Monte Carlo simulation

it can be shown that

$$
\begin{aligned}
\mathrm{S}_{\mathrm{rx}}= & e^{-\alpha}\langle\hat{s}|\vec{\sigma}| \hat{s}\rangle+e^{-\alpha}\langle\hat{s}|\hat{\alpha}| \hat{s}\rangle \sinh (\alpha) \\
& +e^{-\alpha}\langle\hat{s}|\hat{\alpha}(\hat{\alpha} \cdot \vec{\sigma})| \hat{s}\rangle \sinh ^{2}\left(\frac{\alpha}{2}\right)
\end{aligned}
$$

For a polarization multiplexed signal the first and third term will average to zero and hence the Stokes vector measured by a polarimeter will be given by

$$
\mathrm{E}\left\{\mathrm{S}_{\mathrm{rx}}\right\}=e^{-\alpha} \mathrm{E}\{\langle\hat{\mathrm{s}}|\hat{\alpha}| \hat{\mathrm{s}}\rangle\} \sinh (\alpha)
$$

when averaged across all signal points noting that we expect the magnitude of $\{\langle\hat{s}|\hat{\alpha}| \hat{s}\rangle\}$ to converge to unity. Hence the degree of polarization (DOP) will be given by

$$
\operatorname{DOP}=\frac{\mathrm{e}^{-\alpha} \sinh (\alpha)}{\mathrm{S}_{0}}
$$

where $S_{0}=E\left\{\left\langle\mathrm{~T}_{\mathrm{PDL}} \hat{\mathrm{s}} \mid \mathrm{T}_{\mathrm{PDL}} \hat{\mathrm{s}}\right\rangle\right\}$. We note that

$$
\begin{aligned}
\left\langle\mathrm{T}_{\mathrm{PDL}} \mathrm{s} \mid \mathrm{T}_{\mathrm{PDL}} \mathrm{s}\right\rangle & =\left\langle s\left|\mathrm{~T}_{\mathrm{PDL}}^{\mathrm{H}} \mathrm{T}_{\mathrm{PDL}}\right| \mathrm{s}\right\rangle=\left\langle\mathrm{s}\left|\mathrm{T}_{\mathrm{PDL}}^{2}\right| \mathrm{s}\right\rangle \\
& =e^{-\alpha}(\cosh \alpha+\langle\hat{s}|\hat{\alpha} \cdot \vec{\sigma}| \hat{s}\rangle \sinh \alpha)
\end{aligned}
$$

and since a polarization multiplexed signal generates points across the Poincaré sphere the second term will average to zero giving $S_{0}=e^{-\alpha} \cosh \alpha$ and hence the $\mathrm{DOP}=\tanh (\alpha)$. Finally we recall that $\mathrm{PDL}_{\mathrm{dB}}=20 \alpha \log _{10}(\mathrm{e})$ we obtain our final expression the PDL in decibels as

$$
\mathrm{PDL}_{\mathrm{dB}}=20 \log _{10}(\mathrm{e}) \times \tanh ^{-1}(\mathrm{DOP})
$$

In order to assess the validity of Eqn. 18 we perform a Monte Carlo simulation based on random generation of PDL, with the PDL and DOP calculated from the resulting Jones and coherency matrix respectively. The results illustrated in Fig. 26, indicate the validity of the Eqn. (18) as a means of estimating PDL from DOP.

\section{ACKNOWLEDGMENTS}

Hou-Man Chin and Seb J. Savory gratefully acknowledge donation of equipment and support from Ciena, in particular from Michel Bélanger and Doug McGhan. Seb J. Savory thanks the RAEng/The Leverhulme Trust for funding his Senior Research Fellowship. Hou-Man Chin also thanks Ciena 
for funding his $\mathrm{PhD}$ studentship and acknowledges support by EU ICONE project grant \#608099. This research was performed under the auspices of a Ciena university collaborative research grant.

\section{REFERENCES}

[1] M. W. Long and J. D. Narciso, "Probabilistic Design Methodology for Composite Aircraft Structures," National Aeronautics and Space Administration, Tech. Rep. June, 1999.

[2] P. Poggiolini, "The GN Model of Non-Linear Propagation in Uncompensated Coherent Optical Systems," J. Lightw. Technol., vol. 30, no. 24, pp. 3857-3879, 2012.

[3] R. J. Essiambre, G. Kramer, P. J. Winzer, G. J. Foschini, and B. Goebel, "Capacity limits of optical fiber networks," J. Lightw. Technol., vol. 28, no. 4, pp. 662-701, Feb 2010.

[4] Parameters and calculation methodologies for reliability and availability of fibre optic systems G.911, ITU-T Std.

[5] ETSI, "ETSI Technical Report 247," Tech. Rep., 1996.

[6] I. Yamashita and K. Kikushimas, "Statistical design method of loss budget for the optical subscriber transmission system," Electron. Comm. Jpn. Part I, pp. 61-69, 1987.

[7] T. Batten, A. Gibbs, and G. Nicholson, "Statistical Design of Long Optical Fiber Systems," J. Lightw. Technol., vol. 7, no. 1, pp. 209-217, 1989.

[8] M. K. Moaveni and M. Shafi, "A Statistical Design Approach for Gigabit-Rate Fiber-Optic Transmission Systems," J. Lightw. Technol., vol. 8, no. 7, pp. 1064-1072, 1990.

[9] E. Suhir, A. Bensoussan, G. Khatibi, and J. Nicolics, "Probabilistic design for reliability in electronics and photonics: Role, significance, attributes, challenges," in 2015 IEEE International Reliability Physics Symposium, April 2015, pp. 5C.1.1-5C.1.13.

[10] H.-M. Chin, B. C. Thomsen, and S. J. Savory, "The effect of intra-link power perturbations on channel performance," in European Conference and Exhibition on Optical Communications, 2015.

[11] S. J. Savory and H.-M. Chin, "Probabilistic Design of Nonlinear Optical Transmission Systems," in Opt. Fiber Commun. Conf., 2016, pp. 5-7.

[12] K. Roberts, S. H. Foo, M. Moyer, M. Hubbard, A. Sinclair, J. Gaudette, and C. Laperle, "High Capacity Transport at 100G and Beyond," J. Lightw. Technol., vol. 33, no. 3, pp. 563-578, 2015.

[13] P. Serena and A. Bononi, "A time-domain extended gaussian noise model," J. Lightw. Technol., vol. 33, no. 7, pp. 1459-1472, 2015.

[14] A. Mecozzi and M. Shtaif, "The statistics of polarization-dependent loss in optical communication systems," Photon. Technol. Lett., vol. 14, no. 3, pp. 313-315, 2002.

[15] M. Kuschnerov and M. Chouayakh, "On the Performance of Coherent Systems in the Presence of Polarization-Dependent Loss for Linear and Maximum Likelihood Receivers," Photon. Technol. Lett, vol. 22, no. 12, pp. 920-922, 2010.

[16] N. Rossi, P. Serena, A. Bononi, and S. Member, "Polarization-Dependent Loss Impact on Coherent Optical Systems in Presence of Fiber Nonlinearity," Photon. Technol. Lett., vol. 26, no. 4, pp. 334-337, 2014.

[17] C. Xie, "Polarization-Dependent Loss Induced Penalties in PDM-QPSK Coherent Optical Communication Systems," in Optical Fiber Communication Conference, 2010, p. OWE6.

[18] H.-M. Chin, D. Charlton, A. Borowiec, C. Laperle, M. O'Sullivan, and S. J. Savory, "Experimental Investigation of the Impact of Distributed Link PDL on a Coherent Transmission System," in European Conference on Optical Communications, no. W.4.P1.SC.5.60, 2016.

[19] O. Gerstel, "Elastic Optical Networking : A New Dawn for the Optical Layer?" IEEE Comms. Mag., pp. 12-20, 2012. 